region" (p. 269). The ethnographical and zoological references in the book show high expert knowledge, but it may be noticed, perhaps with surprise, that on pp. 297 and 298 he accepts the theory of the marine origin of the fauna of Lake Tanganyika.

The illustrations in the book are numerous and excellent, and it is illustrated by two fine maps by has more fully developed an idea that he was first led to enunciate in 1888, after the publication of Lord Kelvin's Baltimore lectures on molecular dynamics. Prof. von Lindemann's method consists, not in deriving an empirical relationship between the wavelengths or frequencies of the spectral lines, but in investigating mathematically the possible waves which

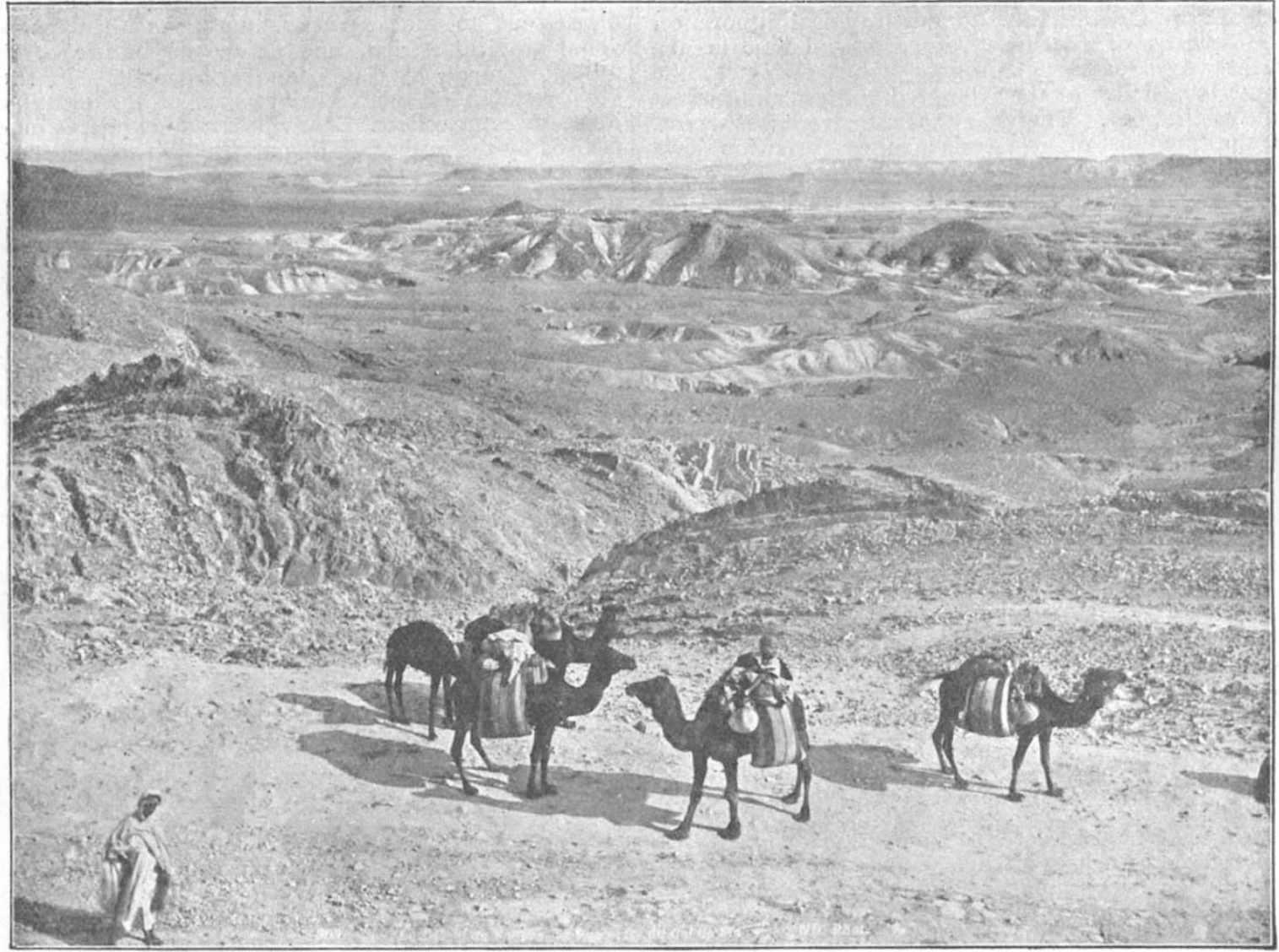

FIG. 2.-In the Libyan Desert. From "The Nie Quest."

Bartholomew, showing the orographic features, and the characteristics of the surface and vegetation in north-eastern Africa.

J. W. G.

\section{THE FORM OF THE ATOMS IN RELATION TO THEIR SPECTRA.}

SINCE Balmer's important discovery in $188_{5}$ that it is possible to calculate the wave-lengths of the first nine lines of the hydrogen spectrum by means of a simple formula, the existence of series of lines, obeying simple mathematical laws, has been established in the case of the spectra of several other elements, notably by the researches of Rydberg and of Kayser and Runge. Among the various attempts that have bet $n$ made to account for these series of lines, and, in general, for the different spectra, the most promising seems to be that of Prof. F. von Lindemann, of Munich, who in some recent papers ${ }^{1}$ 1 "Zur Theorie der Spectrallinien," Sitzingsber. Math. phys. Classen der $K g l$. Bayer. Akad., 1901, xxxi., 441: 1903, xxxiii., 27; a lecture, printed in the Süddeutsche Monatshefte for September, 1905, of which a translation is published in the Monist for January of this year, contains a popular summary of the earlier work and an outline of results not ye pablished in detail. a hypothetical atom can send out into the luminiferous ether.

His assumptions are the simplest possible. His atom consists of a certain amount of elastic isotropic matter of definite shape. The mathematical theory of the different kinds of vibrations of which such a body is capable is well understood, but the actual working out for any special case is difficult because it depends on functions which have to be discovered for each shape, and are, generally speaking, new to mathematicians. The wave-lengths of each kind of vibration sent out into the ether appear always as roots of a transcendental equation involving those functions. Such an equation has an infinite number of roots, each when real corresponding to a definite line. One equation thus corresponds with a " series" of lines. The theory gives for one body a number of such equations, and therefore a number of such "series" of lines, which together form the whole spectrum. This agrees with observed facts.

Prof. von Lindemann investigates, in the first paper quoted, the case of a spherical atom, filled throughout with matter of a definite density and elasticity. In this case, which is comparatively a simple one, the

No. I 895 , vOL. 73] 
calculation can be carried fairly far; but it is found that the spectral lines so deduced obey a law of distribution simpler than any that has yet been found by experiment to characterise any substance. Although atoms are usually assumed for physical calculations to be spherical, such a shape apparently is not really possessed by the atom of any substance; but by using the result established in this case, a simple relationship is shown to be necessary between the wave-lengths of the spectral lines of two similar elements and their atomic weights. If these two elements are conceived as being built up of the same material, having the same form, density, and elasticity, and only their size different, the wave-lengths of corresponding spectral lines of the two elements are shown to be proportional to the cube roots of their atomic weights; given the lines of one of the elements, those of the second element can be calculated from the equation

$$
\frac{\lambda}{\lambda^{\prime}}=\sqrt[3]{\frac{W}{W^{\prime}}},
$$

where $\lambda$ and $\lambda^{\prime}$ are the corresponding wave-lengths, $W$ and $W^{\prime}$ the atomic weights of the two elements. The elements of the following groups are found to obey this rule with a greater or less degree of approximation :-

(I) Zinc, cadmium, and mercury.

(2) Magnesium, calcium, barium, and strontium.

(3) Silver, copper, and gold.

As an illustration, the following series of lines of magnesium and calcium may be given. The arrangement and wave-lengths are those adopted by Kayser and Runge.

\begin{tabular}{|c|c|c|c|c|c|c|c|}
\hline \multicolumn{2}{|c|}{ Magnesium. } & $\mathrm{Mg}=24^{\circ} 4$ & \multicolumn{2}{|r|}{ Calcium. } & \multicolumn{2}{|l|}{$\mathrm{Ca}=40^{\circ} 0}$. & \multirow[b]{2}{*}{$\begin{array}{l}\text { Subor- } \\
\text { diriate } \\
\text { Series }\end{array}$} \\
\hline $\begin{array}{l}\text { Subr. } \\
\text { dinale } \\
\text { Series. }\end{array}$ & $n$ & Observed & Calculated & Observed & Difference & $n$ & \\
\hline I & 4 & $3^{8} 38 \cdot 46$ & $45^{26} \cdot I$ & $45^{27} \cdot 1$ & - I & 一 & - \\
\hline I & 5 & $3097^{\circ} 06$ & $365 \times \cdot 9$ & $\left\{\begin{array}{l}3653^{6} 62 \\
3644.48\end{array}\right.$ & $\begin{array}{r}-172 \\
+7 \cdot 42\end{array}$ & 5 & $\overline{1}$ \\
\hline I & 6 & $2852: 22$ & $3363^{\prime} \mathrm{x}$ & $3361 \cdot 92$ & $\begin{array}{r}7.42 \\
-0.82\end{array}$ & $\begin{array}{l}5 \\
6\end{array}$ & I \\
\hline I & 7 & $2736 \cdot 14$ & $3226 \cdot 2$ & $3225 \cdot 74$ & +0.46 & 7 & I \\
\hline I & 8 & $2673 \cdot 15$ & $3152 \div$ & 3152.08 & -0.08 & 8 & I \\
\hline I & 9 & $2633 \cdot 13$ & $3: 04: 8$ & 3 IOI 87 & +2.93 & 9 & $\mathrm{I}$ \\
\hline I & IO & $2605 \cdot 40$ & $3072 \cdot 1$ & - & + & - & $\longrightarrow$ \\
\hline I I & 3 & $5183 \cdot 84$ & $6102 \cdot 1$ & $6102 \cdot 99$ & -0.80 & - & - \\
\hline I I & 4 & $3336 \cdot 83$ & $3934^{\prime} 5$ & $3933 \cdot 83$ & +0.67 & - & - \\
\hline I I & 5 & $2942 \cdot 21$ & $3477^{\circ} 2$ & $3474^{\circ} 98$ & $+2 \cdot 3^{2}$ & 5 & I I \\
\hline I I & 6 & $2781 \cdot 35$ & $3279 \cdot 8$ & $3274 \cdot 88$ & $+4 \cdot 92$ & 6 & I I \\
\hline I I & 7 & $2698 \cdot 44$ & $3 I 8 I \cdot 8$ & $3 I 8 I \cdot 40$ & +0.40 & 7 & I I \\
\hline I I & 8 & $2649^{\circ} 30$ & 3123.9 & 311774 & $+6 \cdot 16$ & 8 & I I \\
\hline
\end{tabular}

From the similarity of their spectra, the elements in each of the foregoing groups appear to be similarly constructed, and the probability of this is strengthened by the analogy of their chemical properties. On the other hand, chemical analogy does not necessarily imply similarity of form in the elements, as is shown in the case of the alkali metals (lithium, sodium, potassium, rubidium, cæsium); these elements, in spite of their close chemical similarity, do not exhibit the simple relationship connecting wave-length and atomic weight found in the groups already named. Either these elements may be considered as built up of different kinds of matter, or if of the same material as possessing different shapes.

Assuming that matter is uniform, the shape of the atom may be yaried, and instead of the simple sphere the case of an elongated ellipsoid of rotation, formed by revolving an ellipse round its major axis, may be considered.. The mathematical theory shows that the spectral lines of such a luminous ellipsoid depend on three numbers, and that therefore these lines will be capable of arrangement in groups according to three principles. These numbers are obtained as the roots of certain transcendental equations, and are to be calculated from the lengths of the axes of the ellipsoid, its density and elasticity, a calculation, however, which on account of its difficulty is hardly practicable. The first of the three numbers determines a group of corresponding lines, a so-called series; the different possible values of the number determine a certain sequence of such series. The second number determines in each series a subordinate group of lines, and the third number a single definite line in each subgroup: The manner in which this third number enters into the calculation shows; moreover, that the frequencies of the single lines in the subgroups will exhibit among themselves constant differences; differences, that is, depending solely on the nature of the given ellipsoid. A type of distribution of the spectral lines is thus afforded by the theory which corresponds with the well known law of distribution established by Rydberg and by Kayser and Runge in the case of the alkali metals. The atoms of these metals ( $\mathrm{Li}, \mathrm{Na}, \mathrm{K}, \mathrm{Cs}, \mathrm{Rb}$ ) may therefore be considered as elongated ellipsoids of rotation, the axial lengths being fully defined in the case of each element, and different in the different elements.

A flattened ellipsoid of rotation, the so-called spheroid, is by calculation found also to give rise to groups, series, and subgroups, but the law of constant differences is not so generally applicable. The roots of the transcendental equations are, in this case, partly imaginary, so that several groups consist of a single strong line, others of a limited number of lines. Such a grouping is actually found in the case of the metals gold, silver, and copper. Hydrogen is also of this type, its atom probably consisting of a thin, round plate, which is to be considered as the limiting case of a flattened ellipsoid.

In the more general type of ellipsoid, that with three unequal axes, the wave-lengths of the spectral lines also depend on three numbers, defined by certain equations, but in this case the lines cannot be arranged in series and groups, but range over the whole spectrum. Only when the form of the ellipsoid approximates to that of an ellipsoid of rotation will a. few series arise. Such a distribution appears to obtain in the spectra of the alkaline earths (barium, strontium, calcium, and magnesium), that is, with elements lying intermediate in chemical behaviour between the alkalies and ordinary metals; the form here approaches that of the elongated ellipsoid of rotation. With zinc, cadmium, and mercury; the form approximates to the flattened type of the rotation ellipsoid.

Perhaps the most striking consequence of the theory is that which follows from an alteration in the shape of one of the simple ellipsoids of rotation. Such a solid can be imagined as being gradually strained in such a way that it passes into the more general ellipsoid with unequal axes. During such deformation the spectral lines will gradually and continuously change, and the mathematical theory predicates that out of each single line eight others can arise. It appears, indeed, that the Zeemann effect, or the resolution of a single line into two or more other lines under the influence of a magnetic field, is explicable on this hypothesis. It may be observed that the normal triplet which should result according to Zeemann's simple theory does not, as a matter of fact, occur by any means frequently, the arrangement of the resolved lines having been shown by recent work to be of a more complex character than was originally supposed. Such a complexity finds a simple explanation in Prof, von Lindemann's theory of strain.

NO. 1895, VOL. 73] 
Two other types of solids in addition to those already mentioned are susceptible of mathematical treatment, namely, the solids derived by the rotation of a circle round an axis not passing through its centre. When the axis does not cut the circle a ring with a circular section is produced, such as an ordinary finger ring, which is open at the middle. When the axis cuts the circle, a solid, which Lindemann calls a "Wulst" or roll, and resembles in form an orange or an apple--is generated. A particle having the first of these shapes, when rendered luminous, would, according to the mathematical theory, give rise to lines having wave-lengths dependent on four numbers, to each of which a series of values can be given. The kind of spectrum which results can best be explained by imagining the spectrum due to a luminous particle of the elongated ellipsoidal type to be displaced several times in succession, the relative position of the lines being slightly modified in each shift. Such a spectrum has already been found to characterise oxygen and helium; the oxygen spectrum, indeed, according to Runge and Paschen, appears as if derived from that of an alkali metal by a series of successive displacements. An atom of the second type, with a shape similar to that of an apple, when rendered luminous, would, according to the calculations, give rise to a spectrum such as would be produced by successive displacements of the lines due to a flattened ellipsoid. The spectra of sulphur and selenium seem, indeed, to be of this type, being derived from a spectrum like that of oxygen by substituting single strong lines for certain groups of lines. The atom of oxygen thus appears to have the form of an open ring, the atom of sulphur or selenium that of a "Wulst."

Certain interesting consequences concerning the chemical properties of the elements follow from a consideration of their shape, and have been developed by Prof. Lindemann. That the ring-shaped oxygen atom, for example, is a dyad with regard to hydrogen at once follows from the plate-like shape of the hydrogen atoms, two of these being necessary to close the two apertures of the ring. A distinction, moreover, such as is actually found to exist, is introduced at the outset between valency with regard to hydrogen and valency with regard to oxygen. Apart from speculations of this kind, Prof, von Lindemann's work has great significance at the present moment, in that it demonstrates the possibility to derive those physical constants which most clearly define and characterise the individual elements from the conception of a single kind of matter merely by introducing the idea of shape. It is, of course, possible that the atoms do not possess strictly, but only approximately, the simple shapes which can be treated mathematically. If this were so, slight changes would be introduced into the transcendental equations, and the deduced values, for example those in the table given, can be considered only as a first approximation; but the approximation is sufficiently close to justify the belief that the general type of the transcendental equations is correct.

W. A. D.

\section{THE TIME OF FRANCE.}

A NOTE from the Paris correspondent of a daily journal stating that the proposal to adopt Greenwich time in France is again being brought forward, a desirable reform which would bring our nearest neighbour into harmony. in this respect with almost the whole of Europe, may be considered a sufficient reason for giving some facts on the subject under discussion.

Without going back to the earliest proposals for establishing a time-system which should be common to the whole world, an early stage in the movement was the calling of a conference by the Government of the United States to be held at Washington in October, I884. At this meeting, which was attended by representatives of twenty-five nations, but who, it must be remembered, had no power to bind their Governments to any plan of action, it was resolved that "the Conference proposes to the Governments here represented, the adoption of the meridian passing through the centre of the transit instrument at the Observatory of Greenwich as the initial meridian for longitude." This resolution was voted for by representatives of twenty-two countries, one representative took the opposite view, and two countries, of whom France was one, abstained from voting.

Following on this, a resolution was passed at the meeting adopting the principle of a universal day which should begin at mean midnight of the initial meridian, a scheme containing the germ of the present hourly zone system. But a more practical step had already been taken by the managers of the American railways, who, in November, I883, had adopted the now well-known system in which the American continent is divided into five zones, the time used in each of which is respectively $4,5,6,7$, and 8 hours slow on Greenwich. It says much for the breadth of view of the American railway managers, who thus rose above all consideration of national feeling and selected a zero which was likely to suit the convenience of the greatest number, and set an example which must have done much to forward the scheme.

Since 1884 there has been no open international intercourse on the subject, but gradually the zone time system has made its way. In I892 Belgium and Holland began to use Greenwich time; in 1893 mid-European time, one hour fast on Greenwich, was made the legal standard time in Germany and Italy; in the next year the same time was adopted in Switzerland and Denmark, and in 1895 in Norway, Mid-European time had already been in use in Sweden many years, and on the Austrian, Hungarian, Servian, and Macedonian railways since I89I, but, strangely enough, Vienna, the home of Dr. Schram, who was one of the leaders of the movement for the unification of time, has not adopted any legal standard time. The meridian of Pulkowa happens to be 2 hours I minute east of Greenwich, and since the time of this meridian is used for telegraph work and on the railways of Russia, it may be considered that this country uses east European time, two hours fast on Greenwich, which is also used for some purposes in Turkey. Since Greenwich time was made the legal time of Spain in Igoo, it will be seen that almost the whole of Europe has fallen in line. France has not held aloof for want of consideration of its merits. In 1896 the proposition that the Greenwich meridian should be adopted in France was brought by $M$. Deville before the Chamber of Deputies, and being voted on was accepted by that body, but the matter went no further, the reason for which may be inferred from the proceedings at the meeting of the Astronomical Society of France held on December 2, 1896 . At that meeting several of the leading scientific men of France were present, and among them M. Bouquet de la Grye, who, after expressing his astonishment that scientific men had not been consulted before such a proposition was made, proceeded to raise objections. It was true, said he, that the meridian of Greenwich had been chosen as initial because of the greatness of England's seapower; but, he asked, how long would this continue? England's supremacy in this respect might pass away just as had that of other nations, and what then? 